\title{
Farklı uydu sistemi kombinasyonlarının gerçek zamanlı hassas nokta konumlamaya etkisi
}

\author{
The effect of different satellite system combinations on real-time precise point \\ positioning
}

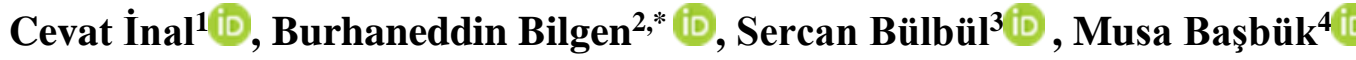 \\ 1, 2, 3,4 Konya Teknik Üniversitesi, Mühendislik ve Doğa Bilimleri Fakültesi, Harita Mühendisliği Bölümü, 42250, Konya, Türkiye
}

\begin{abstract}
Özet
Hassas Nokta Konumlama (PPP) yöntemi, gerçek zamanlı (RT-PPP) ve ölçü sonrası değerlendirme (PP-PPP) olmak üzere iki şekilde uygulanmaktadır. $\mathrm{Bu}$ çalışmada, jeomanyetik aktivitelerin GNSS gözlemleri üzerindeki bozucu etkisinin sonuçlara yansımaması amacıyla Kp, Dst ve F10.7 indisleri incelenmiş ve jeomanyetik aktivitelerin olmadığı 30 ardışık gün belirlenmiştir. Yüksek, orta ve alçak enlem bölgelerinde bulunan Uluslararası GNSS Servisi-Multi GNSS Experiment istasyonlarından KIR8, PADO, NKLG'nin bu ardışık günlere ait Alıcıdan Bağımsız Veri Değişim Formatı (RINEX) verileri RTKLIB v2.4.3 yazılımı ile çözümlenmiştir. Daha sonra, elde edilen kartezyen koordinatlar gerçek değerlerle karşılaştırılmıştır. Ayrıca, her bir istasyona ait koordinat eksenleri yönündeki karesel ortalama hatalar ve konum ortalama hataları hesaplanarak farklı uydu sistemi kombinasyonlarının RTPPP'ye etkisi incelenmiş, genellikle desimetre mertebesinde ortalama hatalar elde edilmiştir. Elde edilen ortalama hatalar değerlendirildiğinde; RT-PPP yönteminin jeodezik uygulamalardan beklenen doğruluğu henüz sağlayamadığı görülmekte, fakat gelecekte hem alıcı hem de antenlerin RT-PPP'yi destekler hâle gelmesi ve yayınlanan gerçek zamanlı düzeltmelerin iyileştirilmesiyle jeodezik uygulamalar için gerekli doğruluğu sağlayabileceği beklenmektedir.
\end{abstract}

Anahtar kelimeler: MGEX, Multi-GNSS, PPP, RT-PPP, RTKLIB

\section{Giriș}

Başlangıçta jeodezik nokta konumlarının belirlenmesinde sadece klasik yersel teknikler kullanılmaktayken, teknolojinin gelişmesiyle birlikte özellikle 1978 yılında Amerika Birleşik Devletleri (ABD) Savuna Bakanlığı'nın askeri amaçlı çalışmalar için ürettiği Küresel Konum Belirleme Sistemi'nin (GPS) hizmete girmesiyle konum belirleme farklı bir boyut ve hiz kazanmıştır. GPS her ne kadar başlangıçta askeri amaçlı ortaya çıkmış olsa da daha sonraları tüm dünyanın hizmetine sunulmuş ve sivil kullanıma izin verilmiştir. ABD'nin bu çalışmalarının yanı sıra özellikle Avrupa Birliği ülkeleri, Çin, Japonya, Rusya ve Hindistan kendi navigasyon

\begin{abstract}
Precise Point Positioning (PPP) method is applied in two ways: real-time (RT-PPP) and post-process (PP-PPP). In this study, Kp, Dst and F10.7 indices were examined in order not to reflect the disturbing effect of geomagnetic activities on GNSS observations on the results, and 30 consecutive days without geomagnetic activities were determined. Receiver Independent Exchange Format (RINEX) data of KIR8, PADO, NKLG which are International GNSS Service-Multi GNSS Experiment stations located in high-, mid- and low-latitude regions processed with RTKLIB v2.4.3 software. Then, the obtained cartesian coordinates were compared with the actual values. In addition, the effect of different satellite system combinations on RT-PPP was examined by calculating both the root mean square errors (RMSE) in the direction of the coordinate axes and 3D RMSE of each station. Generally, the RMSE in decimeter $(\mathrm{dm})$ level were obtained. When the obtained RMSE are evaluated, the RTPPP method has not yet provided the accuracy desired from geodetic applications. However, in the future, it is expected that both the receivers and antennas will support RT-PPP and with the improvement of broadcasted real-time corrections, it will provide the necessary accuracy for geodetic applications.
\end{abstract}

Keywords: MGEX, Multi-GNSS, PPP, RT-PPP, RTKLIB

uydularını yaparak bölgesel/global konum belirleme sistemlerini kurmuşlardır.

Günümüzde aktif olarak hizmet veren Uydularla Konum Belirleme Sistemleri (GNSS); GPS (ABD), GLONASS (Rusya), Galileo (Avrupa Birliği), BEIDOU/COMPASS (Çin), QZSS (Japonya) ve IRNSS/GAGAN (Hindistan) olarak siralanabilir. Tüm bu sistemler bir arada kullanılarak gerçekleştirilen konum belirleme uygulamaları da Çoklu GNSS veya Multi-GNSS gözlemleriyle konum belirleme olarak tanımlanmaktadır. GNSS ile konum belirleme yöntemleri, mutlak konum belirleme ve bağıl (göreli) konum belirleme olarak iki ana başlıkta incelenebilir. Mutlak konum belirlemede tek bir alıcı ile dört ya da daha fazla uydudan

\footnotetext{
* Sorumlu yazar / Corresponding author, e-posta / e-mail: bbilgen @ktun.edu.tr (B. Bilgen)

Geliș / Recieved: 15.09.2021 Kabul / Accepted: 14.10.2021 Yayımlanma / Published: 14.01.2022

doi: $10.28948 /$ ngmuh. 996018
} 
kod/faz gözlemleri yapılarak alıcının kurulu olduğu noktanın koordinatları uzayda geriden kestirme esasına göre hesaplanmaktadır. Bağıl konum belirleme ise, en az iki alıcıdan biri koordinatı bilinen referans noktasına diğeri de koordinatı hesaplanacak olan noktaya kurularak en az 4 uydudan eş zamanlı kod/faz gözlemleri yapılarak koordinatı bilinmeyen noktanın koordinatının hesaplanmasına dayanmaktadır.

Mutlak konum belirleme yöntemlerinden biri olan Hassas Nokta Konumlama (PPP), 1997 yilinda ilk defa Zumberge vd. [1] tarafindan kod ve taşıyıcı faz gözlemleri birlikte kullanılarak tek GNSS alıcısıyla gerçekleștirilmiş bir yöntemdir. PPP, gerçek zamanlı (RT-PPP) ve ölçü sonrası değerlendirme (PP-PPP) olmak üzere iki şekilde uygulanmaktadır. PPP'de hem kod hem de taşıyıcı faz gözlemleri birlikte kullanılırken diğer mutlak konum belirleme yöntemlerinde sadece kod gözlemleri kullanılmaktadır. Ayrıca PPP'de hassas uydu yörünge/saat bilgileri de kullanılmaktadır. Bu yöntemde ölçü süresi ve kullanılan alıcının tek ya da çok frekanslı olması durumuna göre metre $(\mathrm{m})$ ile santimetre $(\mathrm{cm})$ mertebesinde konum doğruluğu elde edilebilmektedir [1-5].

Uluslararası GNSS Servisi (IGS) 2013 yılından itibaren GNSS kullanıcılarına gerçek zamanlı servis hizmeti sunmaya başlamıştır. Böylece kullanıcıların RT-PPP uygulamalarında kullanılan yayın efemerisi, GNSS uydu yörünge ve saat düzeltme verilerine erişimi sağlanmıştır. $\mathrm{Bu}$ sayede, geliştirilen özel yazılımlar kullanılarak RT-PPP yöntemiyle konum belirleme uygulamaları gerçekleştirilebilmektedir.

Günümüzde, RT-PPP yöntemi güncel araştırma konusu olarak dikkat çekmekte ve bu konuda pek çok araştırma yapılmaktadır [6-17]. Gerçekleştirilen çalışmalarda; RT-PPP yönteminin yakınsama süresi, konum doğruluğu ve hassasiyeti, kinematik PP-PPP ile kıyaslanması gibi konular araştırılmıştır. $\mathrm{Bu}$ çalışmada, bugüne kadar yapılan çalışmalardan farklı olarak, yüksek, orta ve alçak enlem bölgelerinde farklı uydu sistemi kombinasyonlarının (GPS, GPS+GLONASS, GPS+Galileo, GPS+BEIDOU, GPS+GLONASS+Galileo+BEIDOU) RT-PPP'ye etkisi RTKLIB v2.4.3 yazılımı ile araştırılmıştır. Bu kapsamda, jeomanyetik aktivitelerin GNSS gözlemleri üzerindeki bozucu etkisinin sonuçlara yansımaması amacıyla Kp, Dst ve F10.7 indeksleri incelenmiş ve jeomanyetik aktivitelerin olmadığı 30 ardışı gün belirlenmiştir. Farklı enlem bölgelerinde bulunan Uluslararası GNSS Servisi-Multi GNSS Experiment (IGS-MGEX) istasyonlarından KIR8, PADO, NKLG'nin bu ardışık günlere ait Alıcıdan Bağımsız Veri Değişim Formatı (RINEX) verileri RTKLIB v2.4.3 yazılımı ile çözümlenmiştir. Çözümlemede yayınlanan gerçek zamanlı yörünge/saat düzeltmeleri (CLK93) kullanılmıştır. Farklı uydu sistemi kombinasyonları ile offline modda RT-PPP çözümü yapılarak elde edilen 30 günlük kartezyen koordinatlar gerçek değerlerle karşıllaştırılmış ve koordinat eksenleri yönündeki karesel ortalama hataları $(\mathrm{KOH})$ ve konum ortalama hataları belirlenmiştir.

\section{Gerçek zamanlı PPP yöntemi ve RTKLIB yazılımı}

IGS tarafindan gerçek zamanlı hassas yörünge ve saat düzeltme veri akışlarının kullanıma sunulmasıyla, kullanıcılar için tek alternatif gibi görünen ölçü sonrasındaki değerlendirme işlemi zorunluluk dışında artık sadece bir tercih olarak kullanılmaktadır. RT-PPP yönteminde tam sayı belirsizlik çözümünün yanı sıra uydu sayısı ve uydu geometrisi yöntemin doğruluğunu etkilemektedir. GPS uydularıyla birlikte GLONASS, Galileo, BEIDOU uydu saat ve yörünge düzeltmelerinin (CLK93) de Uzay Çalışmaları Ulusal Merkezi (CNES) tarafindan yayınlanmasıyla, RTPPP uygulamalarında konum doğruluğunu artırmak ve yakınsama süresini azaltmak amacıyla dört uydu sistemi birlikte değerlendirilebilmektedir. Her ne kadar sistemlerin birlikte kullanılmasının birçok avantajı olsa da sistemler arasındaki farklıliktan kaynaklanan problemler konum doğruluğunu olumsuz etkileyebilmektedir.

RTKLIB yazılımı açık kaynak kodlu (open-source and free software) bir GNSS veri işleme yazılımıdır. RTKLIB Tokyo Üniversitesi Deniz Bilimi ve Teknolojisi bölümü (Tokyo Univ. of Marine Science and Technology) tarafindan geliştirilmiş̧ir. Gerçek zamanlı ve ölçü sonrası değerlendirme şeklinde çeşitli GNSS uygulamalarını (statik, kinematik, DGNSS, PPP vb.) gerçekleştirebilmektedir. GPS, GLONASS, Galileo, QZSS, BEIDOU ve SBAS'a ait veriler RTKLIB yazılımında işlenebilmektedir. Ayrıca yazılım standart GNSS veri protokollerini (RINEX, NMEA, RTCM) desteklemektedir. Yazılımla ilgili ayrıntılı bilgi Takasu [18] tarafindan verilmiştir.

\section{GNSS gözlemlerini etkileyen jeomanyetik aktiviteler}

Kp indeksi, Dst indeksi, güneş patlaması ve güneş akısı değerleri iyonosferdeki değişimlerin büyüklüğünü yansıtan kriterlerdir. GNSS veri sonuçlarının yorumlanmasında bu kriterler çok önemlidir [19-22].

$\mathrm{Kp}$ indeksi, güneş parçacıklarının radyasyonunu manyetik etkileriyle ölçmek için tasarlanmıştır. 1932'den beri, Uluslararası Jeomanyetizma ve Havacılık Derneği (IAGA) bünyesinde faaliyet gösteren Uluslararası Jeomanyetik İndeksler Servisi (ISGI) gibi kuruluşlar tarafindan üç saatlik aralıklarla üretilmektedir [23]. Kullanılan veriler dünyanın orta enlem bölgesinde yer alan 13 istasyondan elde edilmektedir [19]. Manyetik alanın etkilerindeki farklılıklara göre Kp indeksi 0-9 arasında değerler almaktadır [19, 24-25].

Dst, iyonosfer tabakasındaki değişiklikleri, manyetik firtınaları ve derecelerini gösteren bir indekstir [19]. Dünya çapında 4 farklı gözlemevinden 1 saatlik zaman aralığında elde edilmektedir (http://wdc.kugi.kyotou.ac.jp/dstdir/dst2/ on Dstindex.html). Kp indeksi ve Dst indeksinin manyetik firtına dereceleri Tablo 1'de gösterilmiştir. Güneş Akısı Kriteri (F10.7) ise iyonosfer tabakasındaki değişikliklerin gözlemlenmesinde dalga boyu $10.7 \mathrm{~cm}$ olan bir dalga vasıtasıyla güneş akısı ölçümleri ile belirlenebilmektedir.

Tablo 1. Kp ve Dst endekslerinin manyetik firtına dereceleri

\begin{tabular}{ccc}
\hline Manyetik firtına dereceleri & Kp indeksi & \multicolumn{1}{c}{ Dst indeksi } \\
\hline G1 Hafif & 5 & Dst $>-20$ \\
G2 Orta kuvvetli & 6 & $-20>$ Dst $>-50$ \\
G3 Kuvvetli & 7 & $-50>$ Dst $>-100$ \\
G4 Çok kuvvetli & 8 & $-100>$ Dst $>-300$ \\
G5 Aşırı & 9 & $-300>$ Dst \\
\hline
\end{tabular}




\section{Uygulama}

Farklı uydu sistemi kombinasyonlarının RT-PPP'ye etkisinin araştııılmasında, jeomanyetik aktivitelerin GNSS gözlemleri üzerindeki bozucu etkisinin sonuçlara yansımamas1 amaciyla Kp, Dst ve F10.7 indeksleri incelenmiş ve jeomanyetik aktivitelerin hafif ve kısmen orta seviyede olduğu 30 ardışı gün (1-30 Eylül 2020) belirlenmiştir (Bk. Şekil 1). Kp, Dst ve F10.7 indeksleri https://omniweb.gsfc.nasa.gov/form/dx1.html web adresinden temin edilmiştir. Yüksek enlem bölgesinde KIR8, orta enlem bölgesinde PADO ve alçak enlem bölgesindeki NKLG istasyonlarının RINEX verileri temin edilmiş, CNES tarafindan yayınlanan uydu saat ve yörünge düzeltmeleri (CLK93) kaydedilmiştir (Bk. Tablo 2).
Şekil 1, Kp indeksinin $0 \mathrm{nt}$ ile $5.7 \mathrm{nt}$, Dst indeksinin -57 nt ile 14 nt ve F10.7 indeksinin 69.5 nt ile 74.4 nt arasinda değiştiğini göstermektedir. Tablo 1 ve Şekil 1 birlikte değerlendirildiğinde, $\mathrm{Kp}$ ve Dst indekslerinin hafif ve kısmen orta seviyede olduğu, F10.7 indeksinin ise çok büyük bir dalgalanma göstermediği görülmektedir.

Kullanılan IGS-MGEX istasyonlarına ait veriler her bir gün için GPS (G), GPS+GLONASS (G+R), GPS+ Galileo $(\mathrm{G}+\mathrm{E}), \quad \mathrm{GPS}+\mathrm{BEIDOU} \quad(\mathrm{G}+\mathrm{C})$, GPS+GLONASS+Galileo+BEIDOU $\quad(\mathrm{G}+\mathrm{R}+\mathrm{E}+\mathrm{C})$ olmak üzere 5 farklı uydu kombinasyonu kullanılarak RTKLIB v2.4.3 yazılımında daha önce kaydedilen CLK93 verisi kullanılarak RT-PPP yöntemiyle off-line modda çözülmüştür.

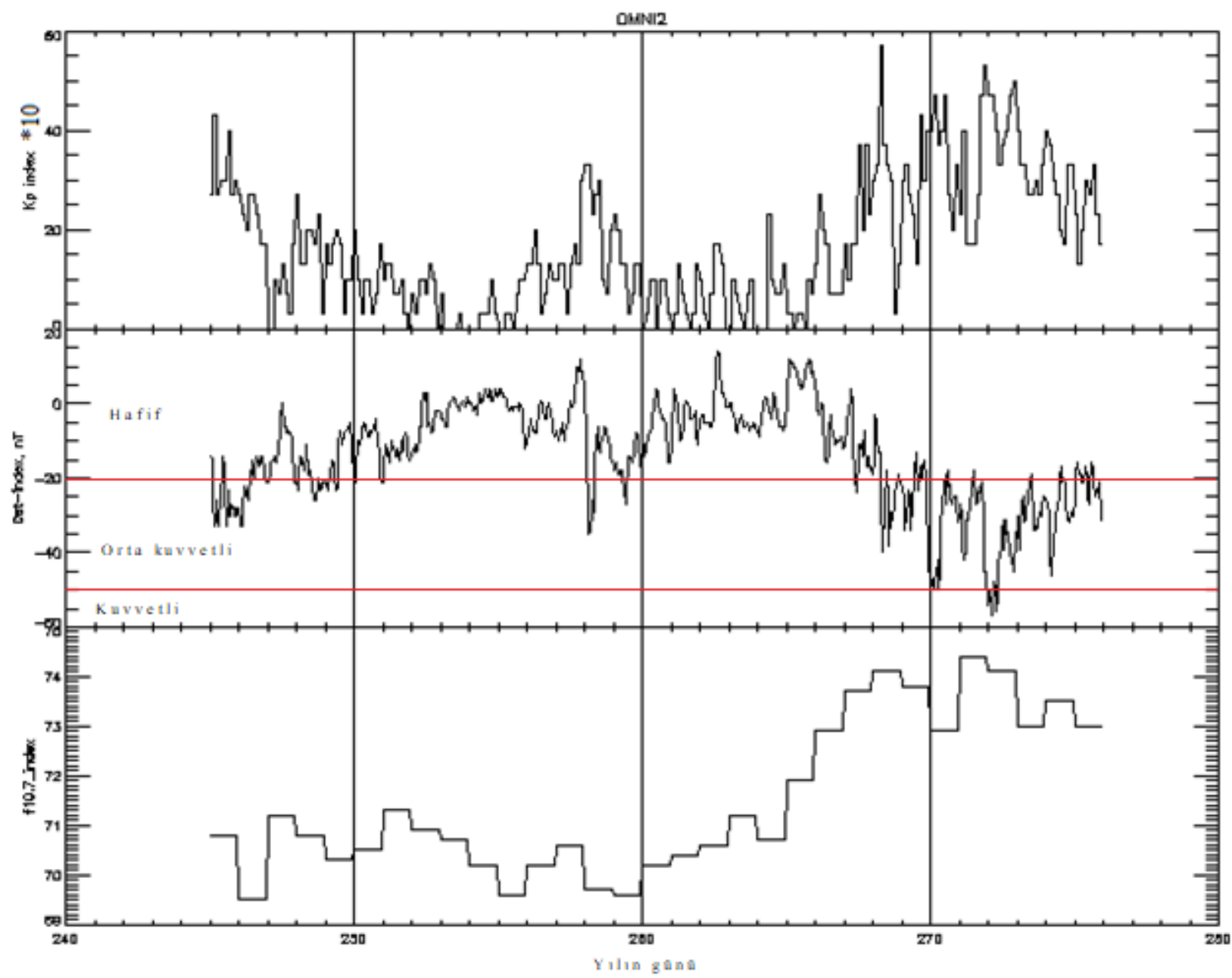

Şekil 1. Ölçü günlerine ait Kp, Dst ve F10.7 indeksleri

Tablo 2. Kullanılan IGS-MGEX istasyonları

\begin{tabular}{cccccc}
\hline Bölge & Nokta Adı & Ülke & Enlem & Boylam & h (m) \\
\hline Yüksek enlem & KIR8 & İsveç & $67^{\circ} 52^{\prime} 39.16^{\prime \prime}$ & $21^{\circ} 03^{\prime} 36.66^{\prime \prime}$ & 498.126 \\
Orta enlem & PADO & İtalya & $45^{\circ} 24^{\prime} 40.15^{\prime \prime}$ & $11^{\circ} 53^{\prime} 45.82^{\prime \prime}$ & 64.699 \\
Alçak enlem & NKLG & Gabon & $00^{\circ} 21^{\prime} 14.07^{\prime \prime}$ & $09^{\circ} 40^{\prime} 19.65^{\prime \prime}$ & 31.501 \\
\hline
\end{tabular}


Tablo 3. IGS-MGEX istasyonlarının 2010.00 epoğunda koordinat ve hızları

\begin{tabular}{ccccccc}
\hline İstasyon & $\mathbf{X}(\mathbf{m})$ & $\mathbf{Y}(\mathbf{m})$ & $\mathbf{Z}(\mathbf{m})$ & $\mathbf{V}_{\mathbf{X}}(\mathbf{m} / \mathbf{y} \mathbf{l})$ & $\mathbf{V}_{\mathbf{Y}}(\mathbf{m} / \mathbf{y} \mathbf{l})$ & $\mathbf{V}_{\mathbf{Z}}(\mathbf{m} / \mathbf{y} \mathbf{l})$ \\
\hline KIR8 & 2248127.0657 & 865685.6020 & 5886424.5526 & -0.01681 & 0.01288 & 0.00453 \\
PADO & 4388881.9292 & 924567.5481 & 4519588.7741 & -0.01620 & 0.01830 & 0.01200 \\
NKLG & 6287385.7445 & 1071574.6406 & 39133.0007 & -0.00416 & 0.02192 & 0.01934 \\
\hline
\end{tabular}

RTKLIB yazılımı RINEX verisi içerisinde yer alan her bir epok için koordinat çözümü gerçekleştirdiğinden, günlük çözümlerin içerisindeki aykırı gözlemler 3 sigma $(3 \sigma)$ kuralına göre ayıklandıktan sonra ortalaması alınarak her bir güne ilişkin nokta koordinatları elde edilmiştir. Noktaların 2010.00 epoğundaki gerçek koordinatları ve yıllık hız bilgileri (http://ftp.aiub.unibe.ch/BSWUSER52/STA/) web sitesinden temin edilmiştir (Bk. Tablo 3).

Gerçek koordinatlar ölçü epoğuna kaydırılarak, RTKLIB yazılımından hesaplanan günlük koordinatlarla karşılaştırması yapılmıştır. Bu amaçla her bir gün için gerçek hatalar;

$\varepsilon_{X_{t}}=X_{t}-X_{G}, \quad \varepsilon_{Y_{t}}=Y_{t}-Y_{G}, \quad \varepsilon_{Z_{t}}=Z_{t}-Z_{G}$

eşitlikleri ile hesaplanmıştır. Burada, altsimge $t$ yılın gününü, altsimge $G$ ise ölçü epoğundaki gerçek koordinatları göstermektedir. Denklem (1)'den hesaplanan gerçek hatalar kullanılarak her bir uydu sistemi kombinasyonu için $\mathrm{KOH}$;

$$
\begin{aligned}
& m_{X}= \pm \sqrt{\frac{\left[\varepsilon_{X_{t}} \varepsilon_{X_{t}}\right]}{n}} \\
& m_{Y}= \pm \sqrt{\frac{\left[\varepsilon_{Y_{t}} \varepsilon_{Y_{t}}\right]}{n}} \\
& m_{Z}= \pm \sqrt{\frac{\left[\varepsilon_{\left.Z_{t} \varepsilon_{Z_{t}}\right]}\right.}{n}}
\end{aligned}
$$

eşitlikleri kullanılarak hesaplanmıştır. Denklem (2)'de $n$ gün sayısını göstermektedir. Bu hesaplamalar her üç istasyonda da 5 farklı uydu kombinasyonu için gerçekleştirilmiştir. İstasyonlara ait $\mathrm{KOH}$ Tablo 4, 5 ve 6'da verilmiştir.

Tablo 4. KIR8 istasyonunda elde edilen $\mathrm{KOH}$

\begin{tabular}{cccc}
\hline Uydu Sistemi & $\boldsymbol{m}_{\boldsymbol{X}}(\mathbf{c m})$ & $\boldsymbol{m}_{\boldsymbol{Y}}(\mathbf{c m})$ & $\boldsymbol{m}_{\boldsymbol{Z}}(\mathbf{c m})$ \\
\hline $\mathrm{G}$ & \pm 10.3 & \pm 5.4 & \pm 1.9 \\
$\mathrm{G}+\mathrm{R}$ & \pm 15.4 & \pm 6.7 & \pm 2.9 \\
$\mathrm{G}+\mathrm{E}$ & \pm 8.9 & \pm 3.4 & \pm 4.4 \\
$\mathrm{G}+\mathrm{C}$ & \pm 27.3 & \pm 11.6 & \pm 1.6 \\
$\mathrm{G}+\mathrm{R}+\mathrm{E}+\mathrm{C}$ & \pm 17.9 & \pm 9.3 & \pm 2.2 \\
\hline
\end{tabular}

Tablo 4, yüksek enlem bölgesinde bulunan KIR8 istasyonuna ait $\mathrm{KOH}$ 'u göstermektedir. KIR8 istasyonunda koordinat bileşenleri yönündeki $\mathrm{KOH} \pm 1.6 \mathrm{~cm}$ ile $\pm 27.3 \mathrm{~cm}$ arasında değişmektedir.
Tablo 5. PADO istasyonunda elde edilen $\mathrm{KOH}$

\begin{tabular}{cccc}
\hline Uydu Sistemi & $\boldsymbol{m}_{\boldsymbol{X}}(\mathbf{c m})$ & $\boldsymbol{m}_{\boldsymbol{Y}}(\mathbf{c m})$ & $\boldsymbol{m}_{\boldsymbol{Z}}(\mathbf{c m})$ \\
\hline $\mathrm{G}$ & \pm 15.7 & \pm 15.1 & \pm 2.4 \\
$\mathrm{G}+\mathrm{R}$ & \pm 18.6 & \pm 10.6 & \pm 2.7 \\
$\mathrm{G}+\mathrm{E}$ & \pm 9.3 & \pm 15.2 & \pm 3.0 \\
$\mathrm{G}+\mathrm{C}$ & \pm 53.9 & \pm 19.8 & \pm 2.6 \\
$\mathrm{G}+\mathrm{R}+\mathrm{E}+\mathrm{C}$ & \pm 29.3 & \pm 9.8 & \pm 3.8 \\
\hline
\end{tabular}

Tablo 5'te orta enlem bölgesindeki PADO istasyonuna ait $\mathrm{KOH}$ görülmektedir. PADO istasyonunda koordinat bileşenleri yönündeki $\mathrm{KOH} \pm 2.4 \mathrm{~cm}$ ile $\pm 53.9 \mathrm{~cm}$ arasında değişmektedir.

Tablo 6. NKLG noktasinda elde edilen $\mathrm{KOH}$

\begin{tabular}{cccc}
\hline Uydu Sistemi & $\boldsymbol{m}_{\boldsymbol{X}}(\mathbf{c m})$ & $\boldsymbol{m}_{\boldsymbol{Y}}(\mathbf{c m})$ & $\boldsymbol{m}_{\boldsymbol{Z}}(\mathbf{c m})$ \\
\hline G & \pm 19.3 & \pm 59.3 & \pm 4.3 \\
$\mathrm{G}+\mathrm{R}$ & \pm 25.8 & \pm 62.3 & \pm 4.0 \\
$\mathrm{G}+\mathrm{E}$ & \pm 12.5 & \pm 56.5 & \pm 2.6 \\
$\mathrm{G}+\mathrm{C}$ & \pm 20.8 & \pm 41.9 & \pm 3.9 \\
$\mathrm{G}+\mathrm{R}+\mathrm{E}+\mathrm{C}$ & \pm 16.5 & \pm 47.5 & \pm 2.6 \\
\hline
\end{tabular}

Tablo 6'da alçak enlem bölgesindeki NKLG istasyonuna ait $\mathrm{KOH}$ sunulmuştur. NKLG istasyonunda koordinat bileşenleri yönündeki $\mathrm{KOH} \pm 2.6 \mathrm{~cm}$ ile $\pm 62.3 \mathrm{~cm}$ arasında değişmektedir. Farklı enlem bölgelerinde $\mathrm{Z}$ ekseni yönündeki KOH'un diğer eksenler yönündeki (X, Y) $\mathrm{KOH}$ 'tan daha iyi olduğu görülmektedir. Farklı enlem bölgelerinde bulunan noktalara ilişkin konum ortalama hatalar1;

$$
m_{p}=\sqrt{m_{X}^{2}+m_{Y}^{2}+m_{Z}^{2}}
$$

Denklem (3) kullanılarak hesaplanmıştır. Hesaplanan konum ortalama hataları Şekil 2'de gösterilmiştir.

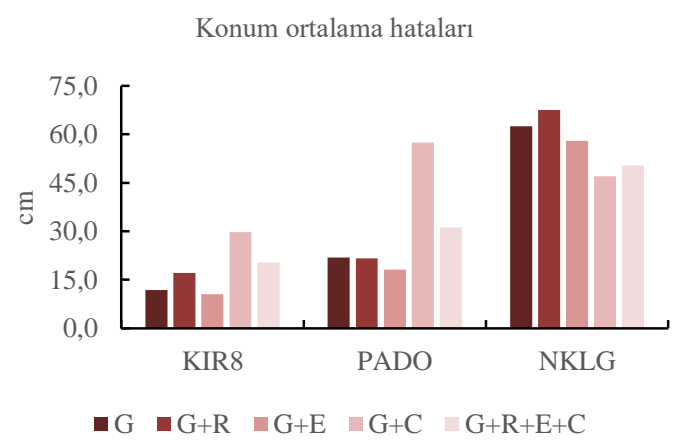

Şekil 2. Konum ortalama hataları 
Tablo 7. Aynı uydu sistemi kombinasyonlarının karşılaştırılması

\begin{tabular}{|c|c|c|c|c|c|c|c|c|c|c|}
\hline \multirow{2}{*}{$\begin{array}{c}\text { Uydu Sistemi } \\
\text { İstasyon }\end{array}$} & \multicolumn{2}{|c|}{ G } & \multicolumn{2}{|c|}{$\mathbf{G}+\mathbf{R}$} & \multicolumn{2}{|c|}{$\mathbf{G}+\mathbf{E}$} & \multicolumn{2}{|c|}{$\mathbf{G}+\mathbf{C}$} & \multicolumn{2}{|c|}{$\mathbf{G}+\mathrm{R}+\mathrm{E}+\mathrm{C}$} \\
\hline & KIR8 & PADO & KIR8 & PADO & KIR8 & PADO & KIR8 & PADO & KIR8 & PADO \\
\hline PADO & $3.459^{*}$ & - & 1.603 & - & $2.965^{*}$ & - & $3.744 *$ & - & $2.353^{*}$ & - \\
\hline NKLG & $28.140 *$ & $8.136^{*}$ & $15.709^{*}$ & $9.800^{*}$ & $30.466^{*}$ & $10.276^{*}$ & $2.497 *$ & 1.499 & $6.157^{*}$ & $2.616^{*}$ \\
\hline
\end{tabular}

Yüksek enlem bölgesi için en iyi konum doğruluğu $\mathrm{G}+\mathrm{E}$ uydu kombinasyonunda elde edilmiş ve bunu sırasıyla $G$, $\mathrm{G}+\mathrm{R}, \mathrm{G}+\mathrm{R}+\mathrm{E}+\mathrm{C}, \mathrm{G}+\mathrm{C}$ kombinasyonları izlemiştir. Orta enlem bölgesi için en iyi konum doğruluğu $\mathrm{G}+\mathrm{E}$ uydu kombinasyonunda elde edilmiş ve bunu sırasıyla $G+R, G$, $\mathrm{G}+\mathrm{R}+\mathrm{E}+\mathrm{C}, \mathrm{G}+\mathrm{C}$ kombinasyonları izlemiştir. Alçak enlem bölgesi için en iyi konum doğruluğu $\mathrm{G}+\mathrm{C}$ uydu kombinasyonunda elde edilmiş ve bunu sırasıyla $\mathrm{G}+\mathrm{R}+\mathrm{E}+\mathrm{C}$, $\mathrm{G}+\mathrm{E}, \mathrm{G}, \mathrm{G}+\mathrm{R}$ kombinasyonları izlemiştir.

Normal dağılımlı iki ölçü grubunun varyanslarının istatistiksel olarak uyuşumlu olup olmadığının test edilmesinde Fisher dağılımı kullanıldığı için, farklı enlem bölgelerinde elde edilen konum ortalama hatalarının istatiksel olarak birbirleri ile karşılaştırılmasında F-testinden yararlanılmıştır. Karşılaştırmada, test büyüklüğü;

$$
F_{\text {test }}=\frac{m_{i}^{2}}{m_{j}^{2}}
$$

eşitliği ile hesaplanmıştır [26]. Test büyüklüğünün hesaplanmasında, büyük olan varyans değeri paya yazılmalıdır. Denklem (4)'te, $m_{i}$ ve $m_{j}$ : i ve j ölçü gruplarının varyanslarını göstermektedir. $f_{i}, f_{j}$ ölçü gruplarının serbestlik dereceleri ve $\alpha$ yanılma ihtimali olmak üzere, $F_{\text {test }}$ değeri $F_{\text {tablo }}=F_{f_{i}, f_{j}, 1-\alpha}$ değeri ile karşılaştırılmıştır. $\quad$ Karşılaştırmalarda; $\quad F_{\text {tablo }}=$ $F_{30,30,0.95}=1.841$ olarak alınmıştır. Farklı enlem bölgelerinde aynı uydu sistemi kombinasyonlarının birbirleri ile karşılaştırılmasında test büyüklükleri hesaplanmış ve Tablo 7'de verilmiştir.

Tablo 7, yüksek enlem ve orta enlem bölgesinde $\mathrm{G}$ ile orta enlem ve alçak enlem bölgesinde $\mathrm{G}+\mathrm{C}$ haricinde, farklı enlem bölgelerinde aynı uydu sistemleri için hesaplanan konum ortalama hatalarının birbirleriyle uyuşumlu olmadığını göstermektedir. Aynı uydu sistemi kombinasyonlarından farklı enlem bölgelerinde elde edilen sonuçlar istatiksel olarak eşit değildir. Farklı enlem bölgelerinde aynı uydu sistemi kombinasyonları karşılaştırıldıktan sonra, aynı enlem bölgelerinde farklı uydu sistemi kombinasyonlarının istatistiksel olarak karşılaştırması yapılmış ve test büyüklükleri Tablo 8, 9 ve 10 'da sunulmuştur. Tablolardaki * işaretiyle anlamlı test büyüklükleri gösterilmiştir.
Tablo 8. Yüksek enlem bölgesinde (KIR8) farklı uydu sistemi kombinasyonlarının karşılaştırılması

\begin{tabular}{cccccc}
\hline $\begin{array}{c}\text { Uydu } \\
\text { Sistemi }\end{array}$ & $\mathrm{G}$ & $\mathrm{G}+\mathrm{R}$ & $\mathrm{G}+\mathrm{E}$ & $\mathrm{G}+\mathrm{C}$ & $\mathrm{G}+\mathrm{R}+\mathrm{E}+\mathrm{C}$ \\
\hline $\mathrm{G}$ & - & $2.092^{*}$ & 1.261 & $6.355^{*}$ & $2.965^{*}$ \\
$\mathrm{G}+\mathrm{R}$ & & - & $2.637^{*}$ & $3.038^{*}$ & 1.418 \\
$\mathrm{G}+\mathrm{E}$ & & & - & $8.012^{*}$ & $3.739^{*}$ \\
$\mathrm{G}+\mathrm{C}$ & & simetrik & & - & $2.143^{*}$ \\
$\mathrm{G}+\mathrm{R}+\mathrm{E}+\mathrm{C}$ & & & & & - \\
\hline
\end{tabular}

Tablo 8'de, G ile G+E'nin ve G+R ile G+R+E+C'nin uyuşumlu olduğu, diğer uydu sistemi kombinasyonları ile hesaplanan konum ortalama hatalarının yüksek enlem bölgesinde istatistiksel olarak eşit olmadığı görülmektedir.

Tablo 9. Orta enlem bölgesinde (PADO) farklı uydu sistemi kombinasyonlarının karşılaştırılması

\begin{tabular}{cccccc}
\hline $\begin{array}{c}\text { Uydu } \\
\text { Sistemi }\end{array}$ & $\mathrm{G}$ & $\mathrm{G}+\mathrm{R}$ & $\mathrm{G}+\mathrm{E}$ & $\mathrm{G}+\mathrm{C}$ & $\mathrm{G}+\mathrm{R}+\mathrm{E}+\mathrm{C}$ \\
\hline $\mathrm{G}$ & - & 1.031 & 1.471 & $6.880^{*}$ & $2.018^{*}$ \\
$\mathrm{G}+\mathrm{R}$ & & - & 1.426 & $7.096^{*}$ & $2.081^{*}$ \\
$\mathrm{G}+\mathrm{E}$ & & & - & $10.119^{*}$ & $2.967^{*}$ \\
$\mathrm{G}+\mathrm{C}$ & & simetrik & & - & $3.410^{*}$ \\
$\mathrm{G}+\mathrm{R}+\mathrm{E}+\mathrm{C}$ & & & & & -
\end{tabular}

Tablo 9 incelendiğinde, orta enlem bölgesinde $\mathrm{C}$ uydu sisteminin konumlamaya dahil edildiği durumlarda hesaplanan konum ortalama hatalarının istatistiksel olarak eşit olmadığı, $G$ ile $G+R$ ve $G+E$ 'nin ayrıca $G+R$ ile $\mathrm{G}+\mathrm{E}$ 'nin uyuşumlu olduğu görülmektedir.

Tablo 10. Alçak enlem bölgesinde (NKLG) farklı uydu sistemi kombinasyonlarının karşılaştırılması

\begin{tabular}{cccccc}
\hline $\begin{array}{c}\text { Uydu } \\
\text { Sistemi }\end{array}$ & $\mathrm{G}$ & $\mathrm{G}+\mathrm{R}$ & $\mathrm{G}+\mathrm{E}$ & $\mathrm{G}+\mathrm{C}$ & $\mathrm{G}+\mathrm{R}+\mathrm{E}+\mathrm{C}$ \\
\hline $\mathrm{G}$ & - & 1.168 & 1.165 & 1.773 & 1.541 \\
$\mathrm{G}+\mathrm{R}$ & & - & 1.360 & $2.071^{*}$ & 1.800 \\
$\mathrm{G}+\mathrm{E}$ & & & - & 1.523 & 1.323 \\
$\mathrm{G}+\mathrm{C}$ & & simetrik & & - & 1.151 \\
$\mathrm{G}+\mathrm{R}+\mathrm{E}+\mathrm{C}$ & & & & & - \\
\hline
\end{tabular}


Tablo 10, alçak enlem bölgesinde $\mathrm{G}+\mathrm{E}$ ve $\mathrm{G}+\mathrm{C}$ ile hesaplanan konum ortalama hataları haricinde, tüm uydu sistemleri ile elde edilen konum ortalama hatalarının istatistiksel olarak uyuşumlu olduğunu göstermektedir.

Kartezyen koordinatlara göre hesaplanan $\mathrm{KOH}$ nokta hızlarındaki hataları da içerdiğinden, bu hataları elemine etmek için günlük olarak elde edilen koordinatların tekrar edilebilirliği incelenmiştir. Bu amaçla, 30 günlük değerlerin ortalamasından sapmalar (STD) hesaplanmış ve Şekil 3'te gösterilmiştir.

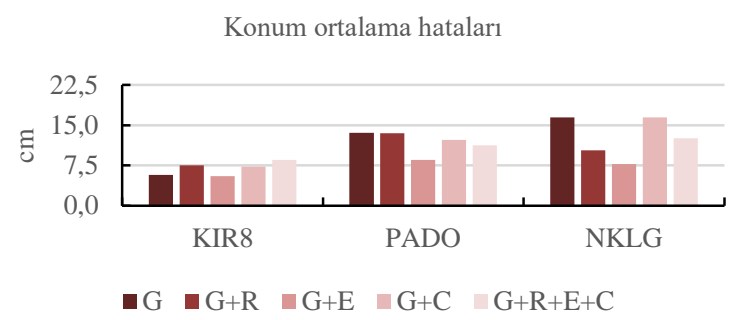

Şekil 3. Konum ortalama hataları

Şekil 3 incelendiğinde, hız bileşenlerine ait hatalar elemine edildikten sonra hesaplanan konum ortalama hatalarının genel olarak \pm 15 cm'nin altında kaldığ görülmektedir. $\mathrm{Bu}$ durum da günlük olarak hesaplanan koordinatların daha tutarlı olarak belirlenebildiğini göstermektedir.

\section{Sonuç ve öneriler}

Günümüzde IGS tarafından gerçek zamanlı yörünge/saat bilgilerinin yayınlanmaya başlamasıyla birlikte bu amaç için geliştirilen yazılımlar kullanılarak RT-PPP yöntemiyle konum belirleme uygulamaları gerçekleştirilebilmektedir. Günümüz itibariyle dört farklı küresel konum belirleme sisteminin tam kapasiteyle kullanımda olması ve bunlara ilişkin hassas yörünge ve saat düzeltmelerinin kullanıcılara sunulması RT-PPP uygulamalarında Multi-GNSS gözlemlerinin etkisinin araştırılmasını ilgi çekici konulardan biri haline getirmiştir. Bu çalışmada; yüksek, orta ve alçak enlem bölgesinde seçilen üç adet IGS-MGEX istasyonu kullanılarak farklı uydu sistemi kombinasyonlarının RTPPP'ye etkisi araştırılmıştır. Bu kapsamda, GNSS ölçüleri üzerinde bozucu etkiye sahip jeomanyetik aktivitelerin fazla olmadığı günler seçilmiştir. Seçilen günlere ait RINEX verileri RTKLIB v2.4.3 yazılımında RT-PPP yöntemi ile beş farklı uydu kombinasyonu kullanılarak off-line modda çözümlenmiştir. Elde edilen koordinatlar gerçek koordinatlarla karşılaştırılmış ve konum ortalama hataları hesaplanmıştır.

Konum ortalama hataları dikkate alındığında; BEIDOU'nun GPS ve diğer uydular üzerinde yüksek ve orta enlemlerde bozucu bir etkiye sahip olduğu görülmüş, fakat GPS uydularına Galileo uyduları eklenince her üç enlem bölgesinde de daha iyi sonuçlar elde edilmiştir. GPS uydularına GLONASS uyduları eklenince yüksek ve alçak enlem bölgesinde bozucu etkiler ortaya çıkmış, orta enlem bölgesinde ise sonuçlar iyileşmiştir. 4 farklı uydu sisteminin birlikte kullanılması durumunda yüksek ve orta enlem bölgelerinde $\mathrm{G}+\mathrm{R}$ ve $\mathrm{G}+\mathrm{E}$ ikili kombinasyonlarına göre daha kötü sonuçlar verdiği görülmektedir. Ancak alçak enlem bölgesinde ise tüm uydu sistemlerinin bir arada kullanılması $\mathrm{G}+\mathrm{R}$ ve $\mathrm{G}+\mathrm{E}$ ikili uydu kombinasyonlarından daha iyi sonuçlar vermiştir. Bunlara ilaveten, farklı enlem bölgeleri bakımından en iyi sonuçlar yüksek enlem bölgesinde elde edilmiş ve bunu orta enlem bölgesi takip etmiştir. Yüksek ve orta enlem bölgelerinde en iyi sonuçlar $\mathrm{G}+\mathrm{E}$ uydu kombinasyonunda elde edilirken, Alçak enlem bölgesinde ise $\mathrm{G}+\mathrm{C}$ uydu kombinasyonunda elde edilmiştir.

Farklı enlem bölgelerinde elde edilen sonuçlar istatistiksel olarak değerlendirildiğinde, aynı uydu sistemi kombinasyonlarında eşit doğruluk elde edilememektedir. Yani, enlem bölgelerinin değişmesi elde edilen konum ortalama hatalarını anlamlı ölçüde değiştirmektedir. Aynı enlem bölgelerinde farklı uydu sistemi kombinasyonlarının istatistiksel olarak karşılaştırılmasında, yüksek enlem bölgesinden alçak enlem bölgesine doğru inildikçe konum ortalama hatalarının birbirleri ile uyuşumlu olma oranlarının arttığı görülmüştür. Dolayısıyla, alçak enlem bölgelerinde tüm uydu sistemi kombinasyonlarında benzer sonuçlar elde edilmiştir.

Gerçekleştirilen çalışma sonucunda; yüksek enlem bölgesindeki $G$ ve $G+E$ kombinasyonları haricinde çoğunlukla dm mertebesinde hatalar elde edilmiştir. $\mathrm{Bu}$ durumda, RT-PPP yönteminin jeodezik uygulamalardan beklenen doğruluğu henüz sağlayamadığı, fakat gelecekte hem alıcı hem de antenlerin RT-PPP'yi destekler hâle gelmesi ve yayınlanan gerçek zamanlı düzeltmelerin de iyileştirilmesiyle jeodezik uygulamalar için gerekli doğruluğu sağlayabileceği düşünülmektedir.

\section{Çıkar çatışması}

Yazarlar çıkar çatışması olmadığını beyan etmektedir.

Benzerlik oranı (iThenticate): $\% 10$

\section{Kaynaklar}

[1] J. F. Zumberge, M. B. Heflin, D. C. Jefferson, M. M. Watkins and F. H. Webb, Precise point positioning for the efficient and robust analysis of GPS data from large networks, Journal of Geophysical Research, 102 (B3), 5005, 1997.

[2] J. Kouba and P. Héroux, Precise point positioning using IGS orbit and clock products. GPS Solutions, 5 (2), 1228, 2001. https://doi.org/10.1007/PL00012883.

[3] Y. Gao and K. Chen, Performance analysis of precise point positioning using Real-Time orbit and clock products, Journal of Global Positioning Systems, 3 (12), 95-100, 2004.

[4] J. Kouba, A guide to using international GNSS service (IGS) products. https://www.igs.org/wp-content/ uploads/2019/08/UsingIGSProductsVer21_cor.pdf , Accessed 10 September 2021.

[5] M. E. Elsobeiey, An improved model for precise point positioning with modernized global positioning system, Master Thesis, Zagazig University, Egypt, 2012.

[6] J. Chen, H. Li, W. Wu, Y. Zhang, J. Wang and C. Hu, Performance of Real-Time precise point positioning, 
Marine Geodesy, $36 \quad$ (1), 98-108, 2013. https://doi.org/10.1080/01490419.2012.699503.

[7] B. Erdoğan, F. Karlıtepe ve T. Öcalan, Farklı IGS kombine yörünge/saat ürünleri (Clk10\&Clk11) kullanarak gerçek zamanlı PPP (RT-PPP) tekniği performansının araştırılması, 8. Ulusal Mühendislik Ölçmeleri Sempozyumu, İstanbul, 2016.

[8] N. Abdi, A. A. Ardalan, R. Karimi and M. H. Rezvani, Performance assessment of Multi-GNSS Real-Time PPP over Iran, Advances in Space Research, 59, 28702879, 2017. https://doi.org/10.1016/j.asr.2017.03.024.

[9] B. Erdoğan, F. Karlıtepe ve T. Öcalan, GPS kinematik PPP'nin gerçek zamanlı PPP (RT-PPP) çözümleri ile karşılaştırılması üzerine bir çalışma, TMMOB Harita ve Kadastro Mühendisleri Odası 16. Türkiye Harita Bilimsel ve Teknik Kurultayı, Ankara, 2017.

[10] F. Karlıtepe, Gerçek zamanlı hassas nokta konumlama (RT-PPP) tekniği ile karşılaştırmalı deneysel çözümler, Yüksek Lisans Tezi, Erciyes Üniversitesi Fen Bilimleri Enstitüsü, Kayseri, 2017.

[11] N. Nadarajah, A. Khodabandeh, K. Wang, M. Choudhury and P. J. G. Teunissen, Multi-GNSS PPPRTK: From large- to smal-scale networks, Sensors, 18 (4) 1078, 2018. https://doi.org/10.3390/s18041078.

[12] M. Turgut, Gerçek zamanlı hassas nokta konumlama (RT-PPP) yönteminin performansının incelenmesi, Yüksek Lisans Tezi, Necmettin Erbakan Üniversitesi Fen Bilimleri Enstitüsü, Konya, 2019.

[13] L. Wang, Z. Li, M. Ge, F. Neitzel, Z. Wang and H. Yuan, Validation and assessment of Multi-GNSS RealTime precise point positioning in simulated kinematic mode using IGS Real-Time service, Remote Sensing, 10 (2), 337, 2018. https://doi.org/10.3390/rs10020337.

[14] S. Xin, X. Li, J. Geng, E. Jiang and Q. Wen, The implementation of multi-GNSS real-time precise point positioning service system: GPS/GLONASS, 2017 Forum on Cooperative Positioning and Service (CPGPS), Harbin, 2017.

[15] S. Alçay, Gerçek zamanlı hassas nokta konumlama (RT-PPP) yönteminin konum belirleme performansının doğruluk ve hassasiyet bakımından incelenmesi, Niğde Ömer Halisdemir Üniversitesi Mühendislik Bilimleri Dergisi, 8 (1), 121-133, 2019. https://doi.org/ 10.28948/ngumuh.516842.

[16] M. Turgut ve S. Alçay, Gerçek zamanlı hassas nokta konumlama (RT-PPP) yönteminin konumlama ve zenit troposferik gecikme (ZTD) bakımından karşılaştırmalı analizi, TMMOB Harita ve Kadastro Mühendisleri Odası 17. Türkiye Harita Bilimsel ve Teknik Kurultayı, Ankara, 2019.

[17] S. Alçay ve Ö. F. Atiz, Farklı yazılımlar kullanılarak gerçek zamanlı hassas nokta konum belirleme (RTPPP) yönteminin performansinın incelenmesi, Geomatik Dergisi, 6 (1), 77-83, 2021. https://doi.org/10.29128/geomatik.687709.

[18] Takasu, T. 2013, RTKLIB 2.4.2 Manual, http://www.rtklib.com/prog/manual_2.4.2.pdf, Accessed 10 September 2021.

[19] N. Arslan, GPS ile iyonosfer toplam elektron yoğunluğu değişimlerinin koordinatlara etkilerinin araştırılması, Doktora Tezi, YTÜ Fen Bilimleri Enstitüsü, İstanbul, 2004.

[20] N. Jakowski, A. Wehrenpfenning, S. Heise, S. Schluter, and T. Noack, Space weather effects in the ionosphere and their impact on positioning, Space Weather Workshop: Looking Towards a Future European Space Weather Programme, Noordwijk, Netherlands, 17-19 December 2001.

[21] P. J. Stewart, and R. B. Langley, Ionospheric modelling for WADGPS at northern latitudes. Proceedings of ION-GPS 98, The 11. International Technical Meeting, 1998.

[22] S. Inyurt, Investigation of ionospheric variations during magnetic storm over Turkey, Geomagnetism and Aeronomy, 60, 131-135, 2020. https://dx.doi.org/ 10.1134/S001679322001012011

[23] S. Inyurt and R. M. Ghaffari, Regional application of ANFIS in ionosphere time series prediction at severe solar activity period, Acta Astronautica, 179, 450-461, 2021.

[24] T. Arslan, M. Shah, S. İnyurt, M. A. Shah, and L. Liu, Comparison of TEC from IRI-2016 and GPS during the low solar activity over Turkey, Astrophysics and Space Science, 365 (11), 2020.

[25] M. Menvielle, The geomagnetic indices: Derivation, meaning and availability, Space Weather Workshop: Looking Towards a Future European Space Weather Programme, Noordwijk, Netherlands, 2001.

[26] C.D. Ghilani, and P.R. Wolf, Adjustment computations: spatial data analysis. Wiley, USA, 2006. 